\title{
The COVID-19 pandemic brings a second wave of social isolation and disrupted services
}

\author{
Alberto Stefana $^{1}$ (1) Eric A. Youngstrom ${ }^{2} \cdot$ Christopher J. Hopwood $^{3} \cdot$ Antonios Dakanalis $^{4}$
}

Received: 31 March 2020 / Accepted: 4 May 2020 / Published online: 15 May 2020

c) Springer-Verlag GmbH Germany, part of Springer Nature 2020

Since December 2019, the outbreak of coronavirus disease 2019 (COVID-19) has infected more than half a million people worldwide [1]. In a short time, governments across the globe decided to place entire cities and nations under quarantine because earlier, milder measures failed to control the COVID-19 spread. Quarantine separates people and restricts the movement of those who may have been exposed to a contagious disease to find out if they become sick and to prevent the disease from spreading.

In this global situation, great attention has immediately focused on the effect of the pandemic on the general population, and in particular, infected patients and frontline responders. Less attention has been given to people with mental health problems [2]. However, our daily work as both public mental health professionals and researchers, sensitizes us to the psychological impact of quarantine on people with mental disorders. Here, we will focus on the Italian situation; however, many other countries now share similar conditions.

Communicated by Andrea Schmitt.

Alberto Stefana

alberto.stefana@gmail.com

Eric A. Youngstrom

eay@unc.edu

Christopher J. Hopwood

chopwoodmsu@gmail.com

Antonios Dakanalis

antonios.dakanalis@unimib.it

1 Department of Clinical and Experimental Sciences, University of Brescia, Viale Europa 11, 25123 Brescia, Italy

2 Department of Psychology and Neuroscience, University of North Carolina at Chapel Hill, CB \#3270, Davie Hall, Chapel Hill, NC 27599-3270, USA

3 Department of Psychology, 268J Young Hall, University of California, 1 Shields Ave, Davis, CA 95616, USA

4 Department of Medicine and Surgery, University of Milano Bicocca, Via Cadore 48, 20900 Monza, Italy
At the time of this writing, Italy is among the countries most affected by the spread of the COVID-19, and has recorded the highest number of deaths (more than 9,000) worldwide [1]. Mass quarantine of the entire country started on March 3 and is scheduled to end on April 3 (although the Italian Government is considering an extension to July 31). It mandates isolation at home (or at community residential facilities) with permitted travel limited to shopping for food, going to work (only for essential services to remain operating), or seeking medical care.

Quarantine has wide-ranging, substantial, and potentially long lasting negative psychological effects on the general population [3], especially when it lasts more than 10 days [4]. Such effects can be even more pervasive and serious in people with mental disorders. Indeed, psychiatric history is a risk factor for experiencing anxiety symptoms and anger 4-6 months after removal from quarantine [5]. Furthermore, history of psychiatric illness is a reliable predictor of posttraumatic stress disorder development in adults exposed to disaster/traumatic-related experiences [6, 7]. Being quarantined involves losing daily routines and personal and social contacts that make life meaningful and provide psychological support, and it comes with substantial costs for individuals with mental illnesses.

The current epidemic situation also significantly disrupts the normal functioning of the Italian public Mental Health Departments: day treatment facilities are closed, home visits are suspended, and mental health outpatient facilities are limiting the visits to patients on depot medication or in psychiatric emergencies. The most symptomatic cases are managed through telephone consultation, and everyone else must wait. Most psychiatric patients cannot regularly attend outpatient visits for evaluation, monitoring, counseling, and prescriptions.

Treatment progress and outcomes suffer when patients are unable to interact with their psychiatrist, psychologist, nurse, or other mental health professionals. The use of quarantine to maintain community health may not be comforting 
for psychiatric patients who are forced to renounce mental health care even as it heightens the need for it.

There is an urgent need to avoid letting strategies being used to mitigate the effects of COVID-19 cause mental suffering in psychiatric patients. Now, more than ever, extra support is needed for this large and vulnerable population. The alternative is asking our patients and their relatives to pay a higher price in term of short- and long-term negative psychological effects, and footing the subsequent-likely massive-bill for the public health system.

The implementation of enhanced psychiatric and psychological intervention services should include, but not be limited to: home visits with social distancing measures in place (when possible), telepsychiatry [8] and telepsychology [9], and the development of telephone and/or video chat support lines staffed by experienced mental health professionals and specifically addressed to quarantined people having difficulties accessing regular mental health services. In all cases of quarantined patients who are completely lacking communication devices, government should provide them access to communication devices [10] to ensure access to these services.

A robust response will save money and avoid the societal risks associated with poor mental health. These efforts will show that we care as a society, and use technology to maintain social connection despite physical distance.

\section{References}

1. Coronavirus COVID-19 Global Cases by the Center for Systems Science and Engineering (CSSE) at Johns Hopkins University (JHU). https://coronavirus.jhu.edu/map.html (accessed 28 Mar 2020)

2. Yao H, Chen JH, Xu YF (2020) Patients with mental health disorders in the COVID-19 epidemic. Lancet Psychiatry 7:e21

3. Brooks SK, Webster RK, Smith LE et al (2020) The psychological impact of quarantine and how to reduce it: rapid review of the evidence. Lancet 395:912-920

4. Hawryluck L, Gold WL, Robinson S et al (2004) SARS control and psychological effects of quarantine, Toronto, Canada. Emerg Infect Dis 10:1206-1212

5. Jeong H, Yim HW, Song YJ et al (2016) Mental health status of people isolated due to Middle East Respiratory Syndrome. Epidemiol Health 38:e2016048

6. Cukor J, Wyka K, Jayasinghe $\mathrm{N}$ et al (2011) Prevalence and predictors of posttraumatic stress symptoms in utility workers deployed to the World Trade Center following the attacks of September 11, 2001. Depress Anxiety 28:210-217

7. Alvarez J, Hunt M (2005) Risk and resilience in canine search and rescue handlers after 9/11. J Trauma Stress 18:497-505

8. American Psychiatric Association. Telepsychiatry. https://www. psychiatry.org/psychiatrists/practice/telepsychiatry (accessed 28 Mar 2020)

9. American Psychological Association. Guidelines for the Practice of Telepsychology. https://www.apa.org/practice/guidelines/telep sychology (accessed 28 Mar 2020)

10. Manuell ME, Cukor J (2011) Mother Nature versus human nature: public compliance with evacuation and quarantine. Disasters $35: 417-442$ 